\title{
Loss of wave-packet coherence in ion-atom collisions
}

\author{
L. Sarkadi* \\ Institute for Nuclear Research of the Hungarian Academy of Sciences (MTA Atomki), P.O. Box 51, H-4001 Debrecen, Hungary \\ I. Fabre, F. Navarrete, and R. O. Barrachina \\ Centro Atómico Bariloche and Instituto Balseiro, Comisión Nacional de Energía Atómica and Universidad Nacional de Cuyo, \\ Avenida Bustillo 9500, 8400 Bariloche, Argentina \\ (Received 25 January 2016; published 3 March 2016)
}

\begin{abstract}
The projectile beam coherence effects occurring in ion-atom collisions are analyzed on the basis of the recent theory of Karlovets et al. [Phys. Rev. A 92, 052703 (2015)] developed for the elastic scattering of wave packets of particles off a potential field. This theory is generalized to estimate the loss of coherence for inelastically scattered projectiles in ionizing collisions. The results obtained by the suggested model are compared with experimental data for the ionization of hydrogen atoms and molecules by $75-\mathrm{keV}$ proton impact. Significantly improved agreement is observed between the theory and experiment.
\end{abstract}

DOI: 10.1103/PhysRevA.93.032702

In a recent work Karlovets et al. [1] investigated theoretically the scattering of wave packets of nonrelativistic particles off a potential field. The authors derived a simple general expression that determines the number of scattering events for the case when the incident particle beam is a wave packet $\Phi$ of arbitrary form but with its mean momentum strongly centered at a given value $\mathbf{p}_{i}$. Furthermore, they considered the example when the wave packet is scattered off randomly distributed potential centers. By averaging the number of events over the impact parameter between the potential center and the wave packet axis, they derived a formula for the effective cross section that reproduces the one obtained by previous authors (see, e.g., $[2,3]$ )

$$
\left.\frac{d \bar{\sigma}}{d \Omega}\right|_{\mathbf{p}_{i} \rightarrow \mathbf{p}_{f}}=\left.\int d \mathbf{k} \frac{k p_{i}}{\mathbf{k} \cdot \mathbf{p}_{i}}|\Phi(\mathbf{k})|^{2} \frac{d \sigma}{d \Omega}\right|_{\mathbf{k} \rightarrow \mathbf{p}_{f}},
$$

where $d \sigma /\left.d \Omega\right|_{\mathbf{q}_{i} \rightarrow \mathbf{q}_{f}}$ is the differential cross section for the elastic scattering in an angle $\theta=\arccos \left(\hat{\mathbf{q}}_{i} \cdot \hat{\mathbf{q}}_{f}\right)$. Furthermore, by writing $\Phi(\mathbf{k})=\Phi_{\perp}\left(\mathbf{k}_{\perp}\right) \Phi_{\|}\left(\mathbf{k}_{\|}\right)$, with $\Phi_{\|}$strongly centered on $\mathbf{p}_{i}$, they obtained

$$
\left.\frac{d \bar{\sigma}}{d \Omega}\right|_{\mathbf{p}_{i} \rightarrow \mathbf{p}_{f}}=\left.\int d \mathbf{k}_{\perp}\left|\Phi_{\perp}\left(\mathbf{k}_{\perp}\right)\right|^{2} \frac{d \sigma}{d \Omega}\right|_{\mathbf{p}_{i}+\mathbf{k}_{\perp} \rightarrow \mathbf{p}_{f}} .
$$

Karlovets et al. carried out sample calculations for the case of electron impact, applying the Born approximation. They considered the collisions of a Gaussian wave packet

$$
\Phi_{\perp}\left(\mathbf{k}_{\perp}\right)=\frac{\sigma_{\perp}}{\sqrt{\pi / 2}} e^{-\left(\mathbf{k}_{\perp} \sigma_{\perp}\right)^{2}}
$$

on a Gaussian potential as well as on the hydrogen atom. Here $\sigma_{\perp}$ is the averaged transverse size of the wave packet. As a remarkable result, they found that the angular distributions of the effective cross section broaden with the decrease of $\sigma_{\perp}$. For example, for collisions of 1.36-keV electrons on hydrogen atoms (in the ground state) the width of the angular distribution of the scattered electrons increases by a factor of $\sim 2.4$ when the plane wave representing the projectile is replaced by a

\footnotetext{
*sarkadil@atomki.hu
}

wave packet with a transverse size $\sigma_{\perp}=0.3$ a.u. (see Fig. 2 in Ref. [1]).

The present work is an attempt to relate the results of Karlovets et al. to those obtained in the past five years in the investigations of the projectile beam coherence effects in ion-atom and ion-molecule collisions [4-13]. It is an interesting question whether the formulas of Karlovets et al. predict a similar wave packet effect in scattering of energetic ions as in the case of electron impact. In their pioneering experiment carried out for the ionization of the $\mathrm{H}_{2}$ molecule by $75-\mathrm{keV}$ protons, Egodapitiya et al. [4] could control the coherence properties of the ion beam and thereby demonstrate the effect of the projectile coherence on the angular distribution of the scattered protons. The principle of the experiment is based on the van Cittert-Zernike theorem according to which the transverse coherence length $\Delta r$ (the diameter of the area of coherence) of a source of waves at a distance $L$ is of the order of $\lambda / k \alpha$, where $\lambda$ is the wavelength, $\alpha$ is the angular diameter of the source, and $k$ is a dimensionless constant.

Egodapitiya et al. used a well-collimated nearly monochromatic $[<1 \mathrm{eV}$ full width at half maximum (FWHM)] proton beam that crossed a molecular hydrogen beam. The measured quantity was the doubly differential cross section (DDCS) for the ionization as a function of the scattering angle $\theta$ and the energy loss $\epsilon$ of the protons. The measurements were made at two coherence length values corresponding to two values of the distance between the target and the last aperture of the collimator, $L=6.5$ and $50 \mathrm{~cm}$. The diameter of the aperture was $a=0.15 \mathrm{~mm}$. Taking $k=1$ in the above expression, it yields $\Delta r=0.86$ a.u. for $L=6.5 \mathrm{~cm}$ and $\Delta r=6.6$ a.u. for $L=50 \mathrm{~cm}$.

A well-known feature of the ionization of diatomic molecules by fast ions is an interference effect that occurs due to the two-center scattering of the projectile. This double-slit interference effect modulates the energy spectrum of the ejected electron [14] and it affects also the angular distribution of the scattered projectile $[15,16]$. For a coherence length smaller than the internuclear separation $D=1.6$ a.u., one does not expect interference effects. Indeed, Egodapitiya et al. found that the interference effect appearing as a dip in the 
angular distribution of the scattered protons was strongly suppressed when the collimator-target distance was changed from 50 to $6.5 \mathrm{~cm}$.

On the basis of their observation, Egodapitiya et al. concluded that "the preparation of the projectile beam affects the scattering cross sections, not because of imperfections in the experiment, but because of the fundamentals of quantum mechanics." In a subsequent work Feagin and Hargreaves [7] arrived at the opposite conclusion: “... experiments with even weakly collimated beams incident on stationary targets cannot distinguish coherent wave-packet structure." The latter authors explained the experimental results of Egodapitiya et al. [4] as follows. According to Feagin and Hargreaves, while Egodapitiya et al. attribute the effect to the low (or high) coherence of the projectile beam, Feagin and Hargreaves disregard the concept of coherence altogether and claim that the same result can be explained as due to a poor (or good) beam collimation. Indeed, by considering the effect of the collimation on the angular resolution for the scattered protons, they obtained reasonable agreement between the experiment and the theory. However, in the experiment the measurements were made with a fixed, good angular resolution $(0.1 \mathrm{mrad}$ FWHM), which is much smaller than the values assumed by Feagin and Hargreaves.

The finding of Egodapitiya et al. that the atomic cross sections can sensitively depend on the projectile coherence was confirmed by further experiments. In these studies, besides the two-center interference occurring in diatomic molecules, further one-center interference effects present also in atomic targets were investigated.

The central idea of the present work is the following. The broadening of the angular distribution with decreasing wavepacket size found by Karlovets et al. [1] in their theoretical work produces an effect similar to that of the decrease of the angular resolution assumed by Feagin and Hargreaves. However, the former effect is an inherent manifestation of the scattering of the wave packet (i.e., it is a real physical effect of quantum origin), while the latter is an artifact of the experiment. It was hoped that by applying the theory of Karlovets et al. [1] for ion impact, the above discrepancy in the interpretation of the experimental findings of Egodapitiya et al. [4] could be resolved.

In the calculation by Karlovets et al., the angle of the scattered electrons in the collisions of $1.36-\mathrm{keV}$ electrons on hydrogen atoms is an order of a few times $0.1 \mathrm{rad}$. For proton impact one expects the angular range to be $m_{p} / m_{e}$ times smaller. Indeed, for the scattering of $75-\mathrm{keV}$ protons on hydrogen atoms [see Eq. (55) in Ref. [1]]

$$
\frac{d \bar{\sigma}}{d \Omega}=f_{0}^{2} \int_{0}^{\infty} \exp (-x g) \frac{x+x^{2}+\left(x^{3} / 6\right)}{1+x / 2 s} d x,
$$

with

$$
g=1+\frac{Q_{\perp}^{2}}{4(1+x / 2 s)}+\frac{Q_{z}^{2}}{4},
$$

results in a cross section that is dominant for scattering angles below $1 \mathrm{mrad}$. Equation (4) is expressed in atomic units and $Q_{\perp}=p_{f} \sin \theta$ and $Q_{z}=p_{f} \cos \theta-p_{i}$ are the transverse and longitudinal momentum transfers, respectively. The dependence on the transverse size of the wave packet

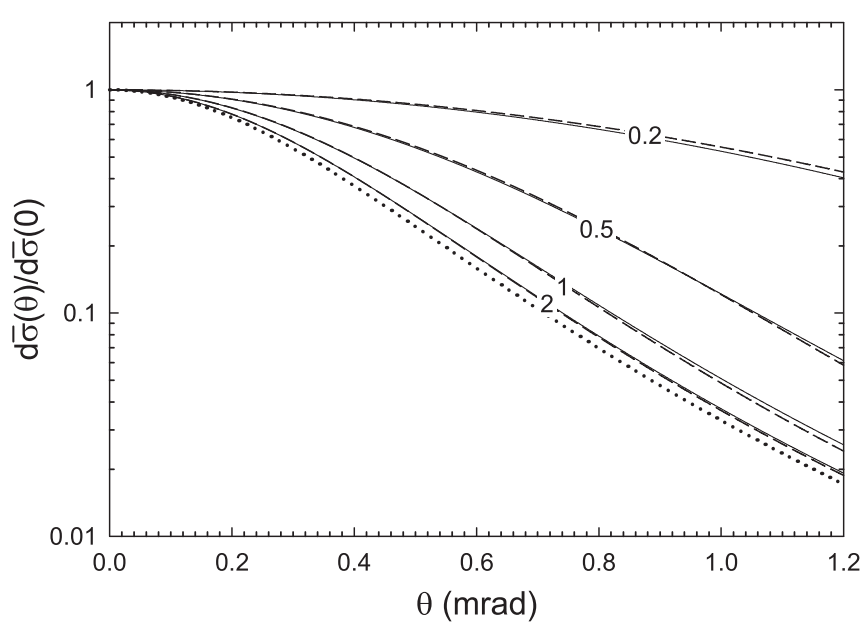

FIG. 1. Normalized angular distribution of the elastically scattered projectiles in collisions of $75 \mathrm{keV}$-protons with hydrogen atoms. The solid lines denote the distributions for wave-packet projectiles with transverse size $\sigma_{\perp}=0.2,0.5,1$, and 2 a.u. [Eq. (4)]; the dotted line represents the solution for plane-wave projectiles [Eq. (5)]; and the dashed lines are results of the approximation given by Eq. (6).

enters the equation through $s=4 \sigma_{\perp}^{2}$. Here $f_{0}$ is a constant whose value is of no interest in the present analysis, since in what follows we deal with the normalized cross section $d \bar{\sigma}(\theta) / d \bar{\sigma}(0)$. At the limit $\sigma_{\perp} \rightarrow \infty$ (plane-wave projectile) the expression (4) yields the standard first Born approximation

$$
\frac{d \bar{\sigma}_{\mathrm{PW}}}{d \Omega}=f_{0}^{2}\left(\frac{1}{g}+\frac{1}{g^{2}}\right)^{2},
$$

with

$$
g=1+\left(Q_{\perp} / 2\right)^{2}+\left(Q_{z} / 2\right)^{2} .
$$

Here the subscript PW denotes plane wave.

Figure 1 shows $d \bar{\sigma}(\theta) / d \bar{\sigma}(0)$ at different values of $\sigma_{\perp}$. As can be seen, the change of the function is small when $\sigma_{\perp}$ decreases from $\infty$ to about 1 a.u. A further decrease of $\sigma_{\perp}$ results in dramatic broadening of the angular distribution. The critical value $\sigma_{\perp}=1$ a.u. can be understood by considering that it is the Bohr radius of the hydrogen atom.

Equation (4) is for elastic scattering. However, due to the linearity of the Schrödinger equation, similar broadening may occur in the angular distribution of the inelastically scattered projectiles. Mathematically, the broadening can be understood considering that Eq. (2) is a convolution of $d \sigma / d \Omega$ with the transverse momentum dispersion $\left|\Phi_{\perp}\left(\mathbf{k}_{\perp}\right)\right|^{2}$ of the wave packet. Therefore, in order to export the broadening effect from the elastic to the inelastic case, we assumed that Eq. (4) can be approximated by a convolution of the plane-wave solution $d \bar{\sigma}_{\mathrm{PW}} / d \Omega$ with a suitably chosen function of the scattering angle that accounts for the broadening. Taking a Gaussian function for the latter, the approximate effective cross section has the form

$$
\frac{d \bar{\sigma}(\theta)}{d \Omega} \approx \frac{1}{\omega \sqrt{2 \pi}} \int_{\theta-\Delta \theta}^{\theta+\Delta \theta} \frac{d \bar{\sigma}_{\mathrm{PW}}\left(\theta^{\prime}\right)}{d \Omega} \exp \left[-\frac{\left(\theta-\theta^{\prime}\right)^{2}}{2 \omega^{2}}\right] d \theta^{\prime} .
$$


Here $\Delta \theta \gg \omega$. With the choice $\Delta \theta=3 \mathrm{mrad}$ we could achieve convergence of the integral for all $\omega$ values considered in the present analysis.

The width $\omega$ of the Gaussian depends on $\sigma_{\perp}$. By fitting the approximate angular distribution (6) to the exact one (4) at several values of $\sigma_{\perp}$, we obtain $\omega=1.69 \times 10^{-4} / \sigma_{\perp}$. Here $\omega$ and $\sigma_{\perp}$ are in units of rad and a.u., respectively. In Fig. 1 we compare the approximate normalized distributions with the exact ones. The agreement is excellent. We note that from Eq. (3) $\omega=1 / 2 p_{i} \sigma_{\perp}$, which for the $75-\mathrm{keV}$ proton yields a slightly smaller value $\omega=1.57 \times 10^{-4} / \sigma_{\perp}$.

Now the loss of coherence in an inelastic collision process can be described by convoluting the theoretical cross section of the process (obtained with plane-wave projectile) with the above convolution function. For the theoretical interpretation of the experimental results of Egodapitiya et al. [4], Feagin and Hargreaves [7] used the DDCS calculated by Chowdhury et al. [17] in the framework of the molecular three-body distorted-wave-eikonal initial-state (M3DW-EIS) approach. This model accounts for the observed interference structure resulting from the two-center potential of the molecule in a way that lower-order approaches, for instance, the first Born approximation, would not achieve [17]. However, it provides too small cross sections. As a correction, in Refs. [7,17] a multiplying factor of 4.5 was applied to the DDCS values. We note that in the present analysis this correction factor is already included in the M3DW-EIS cross sections.

The convoluted cross sections are obtained as

$$
\begin{aligned}
\frac{d^{2} \bar{\sigma}(\theta)}{d \Omega d \epsilon} \approx & \frac{1}{\omega \sqrt{2 \pi}} \int_{\theta-\Delta \theta}^{\theta+\Delta \theta} \frac{d^{2} \sigma_{\mathrm{M} 3 \mathrm{DW}-\mathrm{EIS}}\left(\theta^{\prime}\right)}{d \Omega d \epsilon} \\
& \times \exp \left[-\frac{\left(\theta-\theta^{\prime}\right)^{2}}{2 \omega^{2}}\right] d \theta^{\prime} .
\end{aligned}
$$

We may assume that the transverse size of the wave packet is directly related (i.e., identical) to the coherence length $\sigma_{\perp}=\Delta r$. This assumption can be justified by the definition of the wave packet's transverse size in Ref. [1] as $\sigma_{\perp} \sim 1 / \Delta k_{\perp}$, where $\Delta k_{\perp}$ is the transverse dispersion of the projectile's momentum. Consequently, in the above equation $\omega=1.69 \times 10^{-4} / \Delta r$.

The results of the convolution are plotted in Fig. 2 together with the experimental data [4] and the result of Feagin and Hargreaves [7]. The energy loss of the projectile is $30 \mathrm{eV}$. The experimental cross sections are measured in two series, using partially and practically fully coherent projectile beams. To characterize the degree of coherence, we use the coherence length values 0.86 and 6.6 a.u. We justify this choice to be consistent with the analysis of Feagin and Hargreaves. Here we note that later in the experimental works the coherence length is determined from $\lambda / 2 \alpha$, in which the factor $1 / 2$ is applied because of the special geometry of the beam collimation. Furthermore, it is shown that for the smaller target distance $(L=6.5 \mathrm{~cm})$ the coherence length is not determined sharply; it can take values between 0.43 and 1 a.u.

As can be seen in Fig. 2, the convolution made with $\Delta r=6.6$ a.u. has a negligible effect on the DDCS. For $\Delta r=0.86$ a.u. the effect is significant. For the shape of the angular dependence the convolution in the latter case gives good agreement between the theory and the experiment (with

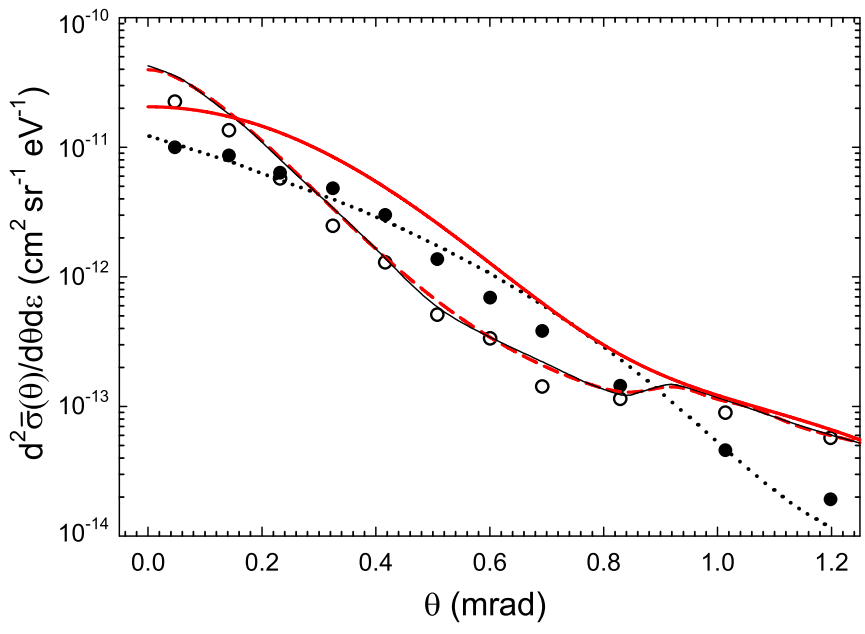

FIG. 2. Comparison of the experimental and theoretical DDCSs for collisions of $75-\mathrm{keV}$ protons with $\mathrm{H}_{2}$ molecules. The symbols represent the experimental data [4]: open circles, $\Delta r=6.6$ a.u.; closed circles, $\Delta r=0.86$ a.u. The thin solid line shows the M3DWEIS theory [17]. The red thick dashed and solid lines are results of the convolution (7) made with $\Delta r=6.6$ and 0.86 a.u., respectively. The dotted line is the result of Feagin and Hargreaves [7] obtained by averaging the M3DW-EIS theoretical data according to Eq. (8).

exception of the two data points above $1 \mathrm{mrad}$ ), though the results overestimate the corresponding experimental data by a factor of about 2. The effective cross section calculated by Feagin and Hargreaves [7] shows better agreement with the experiment. The procedure of these authors is also a kind of convolution, but with a different origin:

$$
\frac{d^{2} \bar{\sigma}(\theta)}{d \Omega d \epsilon} \approx \int_{-\alpha / 2}^{\alpha / 2} \frac{d \theta^{\prime}}{\alpha} \frac{d^{2} \sigma_{\mathrm{M} 3 \mathrm{DW}-\mathrm{EIS}}\left(\theta+\theta^{\prime}\right)}{d \Omega d \epsilon} .
$$

Here $\alpha$ is the convolution width that corresponds to $\omega$ in our procedure. The essential difference between the two procedures is that while $\alpha$ was established by Feagin and Hargreaves from the collimation property of the ion beam $(\alpha=a / L)$, in the present work $\omega$ is related to the coherence length of the beam.

The convolution widths used in the present work and in Ref. [7] are listed in Table I, where the widths are given by the FWHM. While for the Gaussian function in Eq. (7) the FWHM is expressed as $\omega^{2} \sqrt{2 \ln 2}$, the convolution function in Eq. (8) has a rectangular form, therefore the FWHM is directly given by $\alpha$.

Considering that the widths proposed by us are five times smaller that the ones employed in Ref. [7], it is surprising that, according to Fig. 2, the two approaches result in comparable

TABLE I. Convolution widths (FWHM in mrad) used in the present work and in the averaging procedure suggested by Feagin and Hargreaves [7].

\begin{tabular}{lcc}
\hline \hline$L(\mathrm{~cm})$ & Present work & Feagin and Hargreaves [7] \\
\hline 50.0 & 0.060 & 0.3 \\
6.5 & 0.463 & 2.3 \\
\hline
\end{tabular}




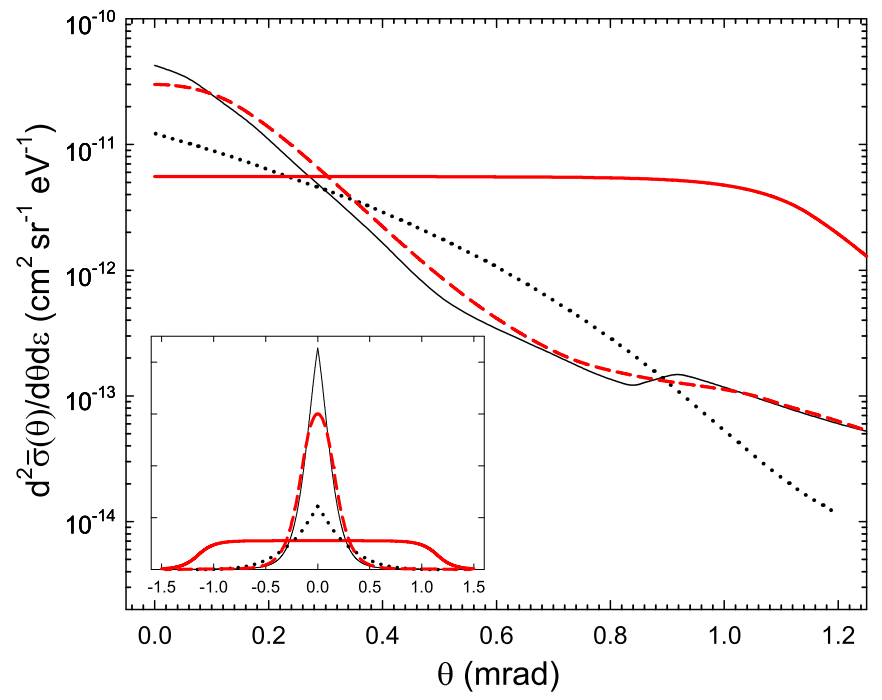

FIG. 3. Results of the convolution (8) evaluated in the present work. The red thick dashed and solid lines are obtained with $\alpha=0.3$ and $2.3 \mathrm{mrad}$, respectively. The thin line shows the M3DWEIS theory [17] and the dotted line is the result of Feagin and Hargreaves [7] for $\alpha=2.3 \mathrm{mrad}$. The inset is a linear plot of the DDCSs in the whole range of the scattering angle in which the convolution (8) is made.

effective cross sections. To resolve this discrepancy, we repeat the convolution expressed by Eq. (8). The results are shown in Fig. 3. For $\alpha=0.3 \mathrm{mrad}$ the DDCS changes only slightly, in accord with the finding of Feagin and Hargreaves. For $\alpha=$ $2.3 \mathrm{mrad}$, however, we obtain a completely different result: The averaged DDCS is constant in almost the whole range of the scattering angle considered in the experiment.

Our result of the constant averaged DDCS can be understood by means of the inset of Fig. 3. As can be seen, for $\alpha=2.3 \mathrm{mrad}$ the range of the integration $[-\alpha / 2, \alpha / 2]$ in Eq. (8) covers almost the whole experimental range of the scattering angle. Compared to this range, the FWHM of the angular distribution of the DDCS predicted by the M3DW-EIS approach is much smaller; it is about $0.24 \mathrm{mrad}$. Considering the latter distribution as a Dirac $\delta$ function, the result of the convolution to a good approximation is the convolution function, which in the present case has a rectangular form with width $\alpha$. It is not clear why the distribution obtained by Feagin and Hargreaves is much narrower than our result.

As mentioned in the introductory part, interference effects may occur not only for diatomic molecules, but also for atomic targets. As an example, we mention the possibility of path interference in atomic collisions discussed by Sarkadi [18]. The author made classical trajectory Monte Carlo (CTMC) calculations to interpret the DDCS measurements of Laforge et al. [19] and Schulz et al. [20] carried out for the ionization of the hydrogen atom by $75-\mathrm{keV}$ proton impact. Comparing also the results obtained with those of several quantum mechanical calculations, he investigated the difference between the classical and the quantum mechanical descriptions of the ionization process by analyzing a large number of classically calculated trajectories of the scattered protons. He found collision events leading to the same final state (energy loss and scattering angle), though belonging to largely different impact parameters. The different impact parameters are due to the different (direct or multiple) scatterings of the projectile, which quantum mechanically can be attributed to first- or higher-order transition amplitudes. The amplitudes of the transitions leading to the same final states are added coherently and as a result constructive or destructive interference may take place. Due to the lack of interference effects in classical mechanics, in Ref. [18] a possible reason for the differences between the CTMC and the quantum mechanical models was attributed to path interference.

The path interference is sensitive to the coherence property of the ion beam. For a beam of small coherence length only collision events belonging to a restricted range of the impact parameter contribute to the scattering cross section, thereby the path interference is suppressed. The above DDCS measurements and the theoretical analysis were made before the beam coherence problem emerged in the field of ion-atom collisions, therefore the role of the beam coherence was not investigated.

The comparison between the CTMC and the quantum mechanical models, however, was not completely conclusive, because while the applied CTMC model described the classical three-body dynamics exactly, the quantum mechanical models were perturbative approaches. From this point of view and also considering the beam coherence problem, the recently developed nonperturbative description of the bare-ion impact-induced ionization of the hydrogen atom [21] is of great significance. The model is based on the coupled pseudostate (CP) formalism, is fully quantal, and includes all the interactions between the particles. It applies both targetand projectile-centered pseudostates, therefore it account for the charge-exchange process as well as the postcollisional interactions between the ionized electron and the two nuclei.

The CP calculations in Ref. [21] were made for $75-\mathrm{keV}$ proton impact. Since in the present work we use the same impact energy, we could check the effect of the loss of the beam coherence on the calculated cross sections. As an example we consider again the DDCS for an energy loss of $30 \mathrm{eV}$. Figure 4 shows the experimental data, the results of the $\mathrm{CP}$ calculations, and the CTMC results. The convoluted theoretical curves evaluated according to Eq. (7) are also plotted. We note that due to a lack of any information about the collimation condition of the proton beam, in the convolution we assume the smallest of the proposed coherence lengths, $\Delta r=0.86$ a.u.

In Fig. 4 the CP calculations are represented by two curves. This is because of an ambiguity in the theory due to the possible double counting of the contributions of the target-centered and projectile-centered continuum states to the ionization cross section. This ambiguity leads to two alternative ways of adding the corresponding ionizations amplitudes, coherently or incoherently.

Let us first consider the theoretical curves before the convolution is applied. As can be seen, the difference between the coherent and incoherent versions is small. Furthermore, the $\mathrm{CP}$ curves support the CTMC result in the sense that they also predict a concave curvature for the scattering angle dependence of the DDCS in contrast to the convex shape shown by the experimental data. This is a surprising result, considering that some of the sophisticated higher-order perturbation models (see, e.g., Refs. [18-20]) predicted a convex shape in accord 


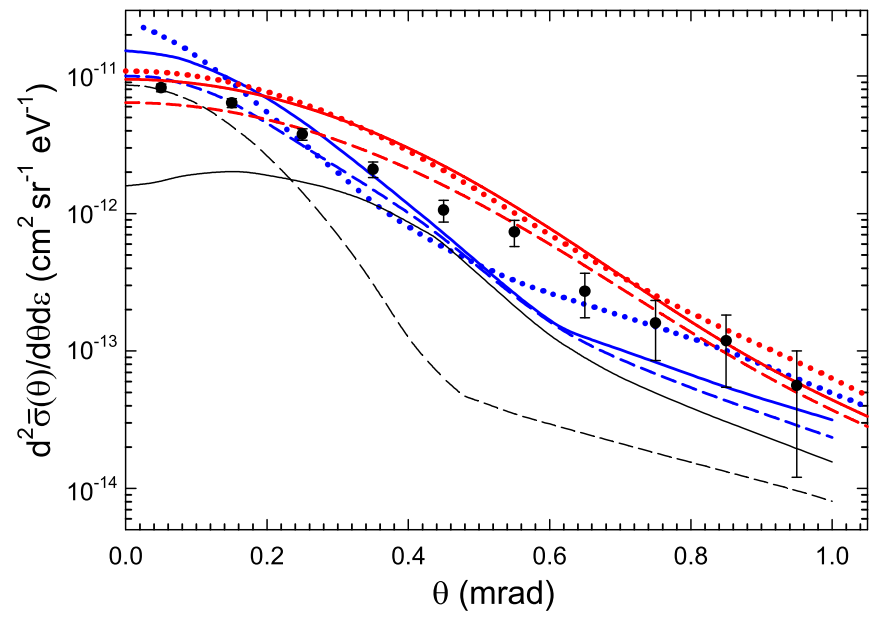

FIG. 4. The DDCS for proton impact ionization of atomic hydrogen at $75 \mathrm{keV}$. The energy loss of the protons is $30 \mathrm{eV}$. The experimental data are from [19,20]. The red (blue) curves are theoretical DDCSs with (without) inclusion of the effect of the loss of the projectile coherence by the convolution (7). The thick solid (dashed) line represents the coherent (incoherent) version of the $\mathrm{CP}$ calculations [21]. The dotted line denotes the CTMC results [18]. The thin black solid (dashed) line shows the contribution of the target- (projectile-) centered pseudostates to the DDCS in the CP calculations.

with the experiment and a similar behavior was expected from even a more realistic and accurate nonperturbative theory. The good qualitative agreement between the CTMC, as a classical approach, and the full quantal nonperturbative $\mathrm{CP}$ model indicates that the path interference effect plays a small role in the scattering process. Otherwise, now the $\mathrm{CP}$ calculations make it clear that the concave shape can be explained by the different scattering angle dependence of the contributions due to the target- and projectile-centered states.

The inclusion of the effect of the loss of the projectile coherence by the convolution (7) changed the character of the theoretical curves from concave to convex. Improved agreement in magnitude with the experiment is also observed. Interestingly, the agreement between the CTMC model and the coherent variant of the $\mathrm{CP}$ calculations is almost perfect. These latter findings make it clear that for a detailed check of the various theoretical models their predictions have to be compared with experimental data obtained with a large beam coherence. We stress that the above analysis was made under the assumption that the transverse coherence length was small in the experiment.
As a conclusion of the present work we can say that we could successfully apply the theory of Karlovets et al. [1] to estimate the effect of the loss of the projectile coherence in ion-atom and ion-molecule collisions. It is important to point out that this approach can also be applied to other scattering processes and specimens besides those analyzed here, even in the case of fully differential cross section (FDCS) measurements. The procedure consists in evaluating an effective cross section for the corresponding elastic scattering of the projectile and target and taking into account the effect of the loss of coherence in the actual process under study by the same convolution method as presented in this work.

Our results support the assessment that the experimentally observed broadening of the angular distribution of the inelastically scattered projectiles with decreasing projectile coherence is an inherent manifestation of the wave-packet scattering and not an experimental artifact. At the same time, as a general property of the wave packet, the broadening effect may smooth any structures appearing in the angular distribution, i.e., not only those that are thought to be related to some interference phenomena. This means that one has to be cautious with the interpretation of the results of the coherence experiments.

Karlovets et al. [1] have also shown that the elastic scattering of the wave packet off the hydrogen atom at nonzero impact parameter leads to azimuthal asymmetry in the angular distributions. The asymmetry strongly depends on the transverse size of the wave packet. This effect may influence the results of FDCS measurements in the field of ion-atom collisions. The dependence of the FDCS on the projectile coherence has already been investigated experimentally $[6,8,10,12,13]$ and theoretically $[11,22]$. One of the goals of the investigations was to solve the so-called $\mathrm{C}^{6+}$ puzzle, a discrepancy between the measured and theoretical FDCSs for the single ionization of $\mathrm{He}$ by $100 \mathrm{MeV} /$ nucleon $\mathrm{C}^{6+}$ projectiles [23], which has remained unexplained for more than a decade. The extension of the present approach to FDCSs on the basis of the theory of Karlovets et al. may solve also the $\mathrm{C}^{6+}$ puzzle. For instance, by the three-dimensional convolution of the inelastic FDCS, the events in the scattering plane (as the binary and recoil peaks) might be partially transferred out of it and also appear in the perpendicular plane in accord with the observation.

This work was supported by the National Scientific Research Foundation (OTKA, Grant No. K109440) and Universidad Nacional de Cuyo (Grant No. 06/C416). I.F., F.N., and R.O.B. also acknowledge support as members of the Consejo Nacional de Investigaciones Científicas y Técnicas (CONICET), Argentina.
[1] D. V. Karlovets, G. L. Kotkin, and V. G. Serbo, Phys. Rev. A 92, 052703 (2015).

[2] E. H. Wichmann, Am. J. Phys. 33, 20 (1965).

[3] J. R. Taylor, Scattering Theory: The Quantum Theory of Nonrelativistic Collisions (Wiley, New York, 1972).

[4] K. N. Egodapitiya, S. Sharma, A. Hasan, A. C. Laforge, D. H. Madison, R. Moshammer, and M. Schulz, Phys. Rev. Lett. 106, 153202 (2011).
[5] S. Sharma, A. Hasan, K. N. Egodapitiya, T. P. Arthanayaka, G. Sakhelashvili, and M. Schulz, Phys. Rev. A 86, 022706 (2012).

[6] X. Wang, K. Schneider, A. LaForge, A. Kelkar, M. Grieser, R. Moshammer, J. Ullrich, M. Schulz, and D. Fischer, J. Phys. B 45, 211001 (2012).

[7] J. M. Feagin and L. Hargreaves, Phys. Rev. A 88, 032705 (2013).

[8] K. Schneider, M. Schulz, X. Wang, A. Kelkar, M. Grieser, C. Krantz, J. Ullrich, R. Moshammer, and D. Fischer, Phys. Rev. Lett. 110, 113201 (2013). 
[9] S. Sharma, T. P. Arthanayaka, A. Hasan, B. R. Lamichhane, J. Remolina, A. Smith, and M. Schulz, Phys. Rev. A 89, 052703 (2014).

[10] S. Sharma, T. P. Arthanayaka, A. Hasan, B. R. Lamichhane, J. Remolina, A. Smith, and M. Schulz, Phys. Rev. A 90, 052710 (2014).

[11] F. Járai-Szabó and L. Nagy, Eur. Phys. J. D 69, 4 (2015).

[12] T. P. Arthanayaka, S. Sharma, B. R. Lamichhane, A. Hasan, J. Remolina, S. Gurung, and M. Schulz, J. Phys. B 48, 071001 (2015).

[13] T. P. Arthanayaka, S. Sharma, B. R. Lamichhane, A. Hasan, J. Remolina, S. Gurung, L. Sarkadi, and M. Schulz, J. Phys. B 48, 175204 (2015).

[14] N. Stolterfoht, B. Sulik, V. Hoffmann, B. Skogvall, J. Y. Chesnel, J. Rangama, F. Frémont, D. Hennecart, A. Cassimi, X. Husson, A. L. Landers, J. A. Tanis, M. E. Galassi, and R. D. Rivarola, Phys. Rev. Lett. 87, 023201 (2001).

[15] L. P. H. Schmidt, S. Schössler, F. Afaneh, M. Schöffler, K. E. Stiebing, H. Schmidt-Böcking, and R. Dörner, Phys. Rev. Lett. 101, 173202 (2008).
[16] J. S. Alexander, A. C. Laforge, A. Hasan, Z. S. Machavariani, M. F. Ciappina, R. D. Rivarola, D. H. Madison, and M. Schulz, Phys. Rev. A 78, 060701(R) (2008).

[17] U. Chowdhury, M. Schulz, and D. H. Madison, Phys. Rev. A 83, 032712 (2011).

[18] L. Sarkadi, Phys. Rev. A 82, 052710 (2010).

[19] A. C. Laforge, K. N. Egodapitiya, J. S. Alexander, A. Hasan, M. F. Ciappina, M. A. Khakoo, and M. Schulz, Phys. Rev. Lett. 103, 053201 (2009).

[20] M. Schulz, A. C. Laforge, K. N. Egodapitiya, J. S. Alexander, A. Hasan, M. F. Ciappina, A. C. Roy, R. Dey, A. Samolov, and A. L. Godunov, Phys. Rev. A 81, 052705 (2010).

[21] H. R. J. Walters and C. T. Whelan, Phys. Rev. A 92, 062712 (2015).

[22] K. A. Kouzakov, S. A. Zaytsev, Y. V. Popov, and M. Takahashi, Phys. Rev. A 86, 032710 (2012).

[23] M. Schulz, R. Moshammer, D. Fischer, H. Kollmus, D. H. Madison, S. Jones, and J. Ullrich, Nature (London) 422, 48 (2003). 